\title{
Effects of dietary oil blend on fatty acid composition, oxidative stability and physicochemical properties of Longissimus thoracis et lumborum muscle in goats
}

\begin{abstract}
This study examined the effects of dietary blend of $80 \%$ canola oil and $20 \%$ palm oil (BCPO) on the physicochemical properties, antioxidant status, oxidative stability and fatty acid composition of Longissimus thoracis et lumborum (LTL) muscle from goats during chill storage. Over a 14-week feeding trial, 24 Boer bucks were randomly assigned to and supplemented with diets containing 0,4 or $8 \%$ BCPO on a dry matter basis, slaughtered and the LTL was subjected to a 7 day chill storage. Neither diet nor post mortem ageing influenced $(\mathrm{P}>0.05)$ antioxidant enzyme activities, chemical composition and cholesterol. Diet had no effect on the carbonyl content, free thiol content, water-holding capacity, tenderness, $\mathrm{pH}$ and glycogen. Oil-supplemented goats had higher $(\mathrm{P}<0.05) \mathrm{C} 18: 1$ trans-11, C18:3n-3 and C20:5n-3, carotenoid, tocopherol and redness, and lower thiobarbituric acid reactive substances values than the control goats. Post mortem ageing decreased $(\mathrm{P}<0.05)$ shear force and oxidative stability of chevon. No significant $(\mathrm{P}>0.05)$ changes were found in the proportion of individual fatty acids throughout storage. Total polyunsaturated fatty acids (PUFA) decreased while total saturated fatty acids increased as storage progressed. Dietary BCPO enhanced n-3 PUFA without compromising the quality attributes of chevon.
\end{abstract}

Keyword: Ageing; Antioxidant; Carbonyl; Cholesterol; Thiol 